\title{
Electronic structure and radial breathing mode for carbon nanotubes with ultra-high curvature
}

\author{
W. Plank', R. Pfeiffer ${ }^{1}$, C. Scharman', L. Wirtz ${ }^{2}$, M. Calvaresi ${ }^{3}$, F. Zerbetto ${ }^{3}$, J. Meyer ${ }^{4}$, and H. Kuzmany ${ }^{*, 1}$ \\ ${ }^{1}$ Fakultät für Physik, Universität Wien, 1090 Wien, Austria \\ ${ }^{2}$ Institute for Electronics, Microelectronics, and Nanotechnology, CNRS-UMR 8520, Department ISEN, \\ 59652 Villeneuve d'Ascq, France \\ ${ }^{3}$ Institute of Inorganic Chemistry, University of Bologna, via F. Selmi, 40126 Bologna, Italy \\ ${ }^{4}$ Universität Ulm, 89081 Ulm, Germany
}

Received 26 May 2010, revised 20 July 2010, accepted 21 July 2010

Published online 14 September 2010

Keywords electronic structure, resonance Raman scattering, ultra-high curvature

*Corresponding author: e-mail hans.kuzmany@univie.ac.at, Phone: +43-1-427751306, Fax: +43-1-427751375

Ultra-high curvature single-walled carbon nanotubes (SWCNTs) with diameters down to $0.37 \mathrm{~nm}$ were prepared by transformation of $\mathrm{FeCp}_{2}$ peapods to double-walled CNTs (DWCNTs). Results from resonance Raman scattering and high resolution transmission electron microscopy (TEM) were compared to calculations on the molecular dynamical, many electron corrected extended tight binding, and density functional theory (DFT) level. The growth process was found to be catalytic from $\mathrm{Fe}_{3} \mathrm{C}$ particles inside the tubes with dimensions of a crystallographic unit cell. The electronic structure showed dramatic deviations from tight binding results. The family behavior leads to level crossing already for $E_{11}$ and $E_{22}$ transitions. Experimental results fitted well into a KatauraPopov plot and allowed assignment for the observed Raman lines of the inner tubes. Experimental and calculated radial breathing mode (RBM) frequencies showed a systematic difference indicating a radial expansion of the smallest inner tubes and a radial compression for the larger tubes.
1 Introduction Single-walled carbon nanotubes (SWCNTs) with ultra-high curvature have recently developed into an attractive research subject from the experimental and from the theoretical point of view. Since special techniques are required to grow such tubes and to evaluate their electronic and vibrational properties these materials may be scheduled into an extra class of SWCNTs. Amongst others the conventional classification of the tubes into metallic and semiconducting (SC) species according to their chiral vector breaks down completely. Other properties such as spin orbit coupling [1,2] or electron-phonon coupling [3] are dramatically enhanced, the latter leading to superconductivity at already moderate temperatures [4].

As a consequence of the strong deviation of these tubes from a planar geometry strong $\sigma-\pi$ mixing is observed. Furthermore, the evaluation of optical spectra must include electron correlation effects and electron-hole interaction $[5,6]$. Good approximations exist for the consideration of such behavior for large and intermediate diameter tubes
$[7,8]$. Even though, experimental and theoretical analysis is very demanding. Fortunately, for the ultra-high curvature tubes the unit cell sizes are strongly reduced due to the short chiral vectors which provides a realistic potential for very high level calculations.

Similarly the evaluation of mechanical and vibrational properties require extension of classical graphene derived descriptions. Parameterized extended tight binding calculations [9] are requested or even density functional theory (DFT) calculations with local-density approximation (LDA) or generalized-gradient approximation (GGA) approximation.

In this contribution, we report on the electronic structure and on the anomalous behavior of the radial breathing mode (RBM) of SWCNTs with ultra-high curvature. Multifrequency resonant Raman scattering is used to determine electronic transition energies and the RBM frequencies for inner tubes in double-walled carbon nanotubes (DWCNTs). We verified with DFT-LDA 
calculations that the RBM frequency of the inner tubes can shift upwards and downwards by several wave numbers relative to the value of the free tube, depending on the radius difference between inner and outer tubes. On average one may therefore compare our experimental results with calculations on the RBM of isolated single-walled nanotubes [10]. In a previous work, Popov and Henrad obtained a similar result using a valence-force field method with an empirical Lenard-Jones potential for the inter-layer carboncarbon interaction [11]. However, they did not perform structural relaxation and therefore could not take into account the effect of a radius variation of the tubes.

As a consequence of the analysis not only dramatic anomalies in the description of the electronic structure but also noticeable deviations from conventional behavior for the RBM frequencies were experimentally recognized. Such behavior of RBM in ultra-high curvature SWCNT is depicted in Fig. 1.

For standard size tubes the frequencies follow a $C / d_{\mathrm{t}}$ law with $C=225$. However, for very small diameter tubes characteristic and quasi-periodic deviations occur for tubes with high chiral angle as depicted in the insert [12]. Since the tubes we are discussing in the following have diameters covering the range depicted in the insert of the figure, experimental investigation of such anomalous behavior was possible for the first time.

2 Experimental procedure and evaluation of RBM frequencies Commercial SWCNTs of HiPco type were filled with ferrocene $\left(\mathrm{FeCp}_{2}\right)$ after the tubes were opened by etching the caps in air at $420^{\circ} \mathrm{C}$. Successful filling

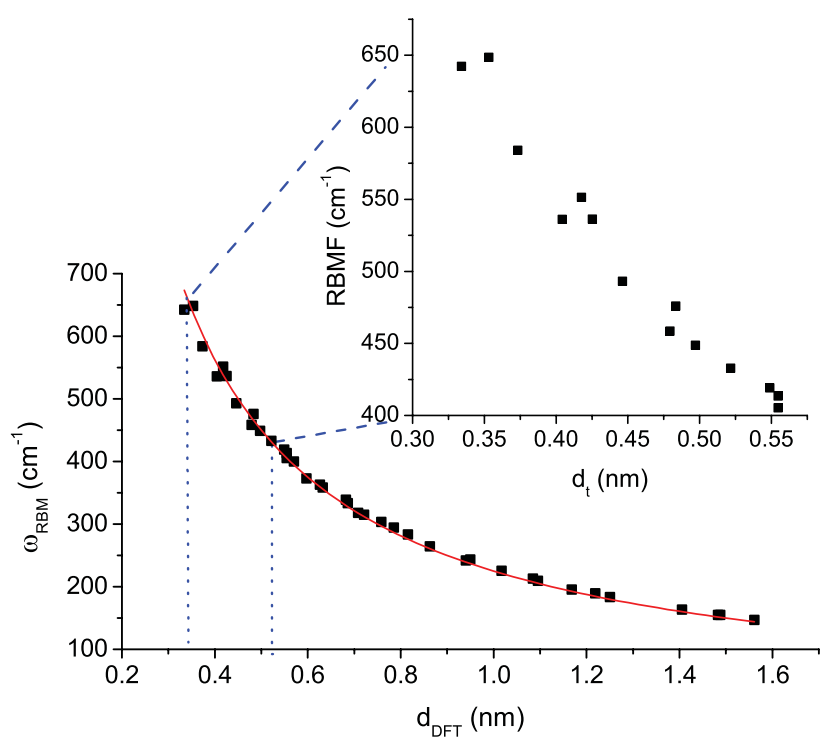

Figure 1 (online colour at: www.pss-b.com) DFT calculated RBM frequencies versus tube diameter, after Kürti et al. [12] The frequencies follow a well defined $1 / d_{\mathrm{t}}$ law for large and moderate small tubes (black squares and full drawn line) but exhibit significant deviation from this behavior for tubes with ultra-high curvature (insert). was monitored by checking the Raman spectra of the resulting ferrocene peapods. Molecular-dynamical calculations provided information that tubes down to a diameter of about $1 \mathrm{~nm}$ will be able to accept the small but carbon rich ferrocene molecule. Transformation to DWCNTs was performed by vacuum annealing at $800^{\circ} \mathrm{C}$ for $2 \mathrm{~h}$ and checked by high resolution TEM. Raman spectra were recorded at $80 \mathrm{~K}$ in the high resolution mode with a large set of laser lines, including tunable systems. In all cases the Raman spectra were recorded before and after the transformation to DWCNTs. These two spectra were subtracted so that the resulting difference spectrum is representative for the response from the inner tubes.

For a selected set of tubes the RBM frequencies were evaluated using DFT calculations within LDA for the handling of the exchange correlation energy as provided in the ABINIT package [13]. For the rest of the frequencies values were used as obtained from the VASP package and reported in Ref. [12]. These values agreed within one wavenumber with the results from the ABINIT package.

3 Results Figure 2 depicts an example for a spectrum recorded with a red laser before and after transformation to DWCNTs. The additional narrow line at $470 \mathrm{~cm}^{-1}$ in the spectrum after annealing represents the response from one special inner tube which is in resonance with the laser used.

The response from the intermediate frequency modes of the outer tubes and their RBM response remain almost unchanged upon the transformation and could therefore be subtracted without problems. This procedure was performed for excitation with 15 different laser lines. A set of 9 resulting Raman lines from the inner tube RBMs is depicted in Fig. 3.

Only lines are considered which appear clearly above the background. The line width is between 6 and $12 \mathrm{~cm}^{-1}$ and thus much higher than the line widths reported for DWCNTs

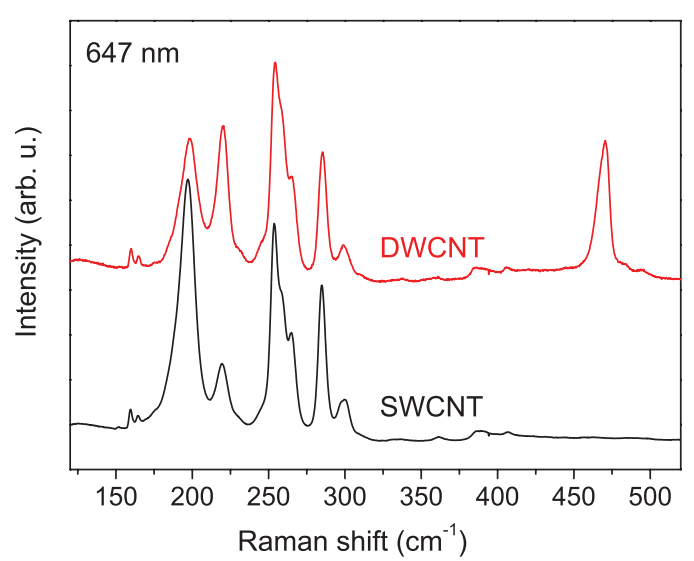

Figure 2 (online colour at: www.pss-b.com) High resolution Raman spectra of HiPco CNTs filled with ferrocene before (bottom) and after (top) transformation to DWCNT. The features on the low frequency side are the RBM response from the outer tubes. The new line at $470 \mathrm{~cm}^{-1}$ is from the inner tube. The background lines originate from outer tubes intermediate frequency modes. 

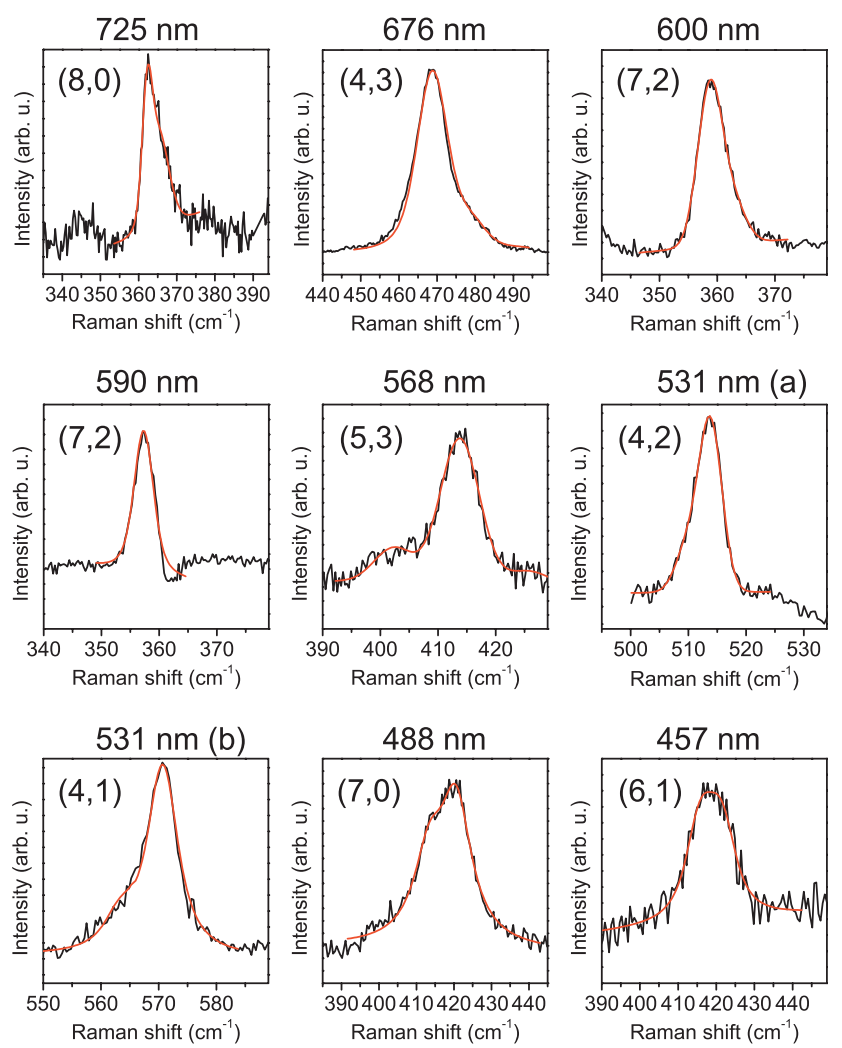

Figure 3 (online colourat: www.pss-b.com) Selected set of Raman lines of inner tube RBMs as obtained after subtraction of the response from the outer tubes. Laser excitation is indicated in $\mathrm{nm}$. The assignment is discussed in Section 3 and summarized in Table 1.

grown from fullerenes in larger diameter tubes [14]. The line shapes exhibit in general a more or less expressed asymmetry which indicates contribution of several components. The lines were in general only observed with one particular laser energy which thus determines the resonance transition energy. From special cases where the resonance was in the energy range of a dye laser, the width of the cross section was found to be only $45 \mathrm{meV}$ which can be considered as the accuracy to which the transition energies were determined.

Knowing the transition energies and the RBM frequencies the experimental results can be inserted into a Kataura plot for transition energies versus RBM frequency. However, since the tubes have ultra-high curvature dramatic deviations from a conventional plot can be expected as they were already observed, albeit with less dramatic, for tubes with a diameter of the order of $1 \mathrm{~nm}[8,15]$. A detailed description for the evaluation of the transition energies for the tubes with ultra-high curvature is reported in Ref. [16]. The slightly modified result is depicted in Fig. 4. The curvature and chirality corrected plot of transition energies versus RBM frequency is now often referred to as KatauraPopov plot. There is family behavior and dramatic deviation from the originally linear relation between transition energies and RBM frequency. Circles and squares correspond to SC and metallic (M) tubes, respectively.

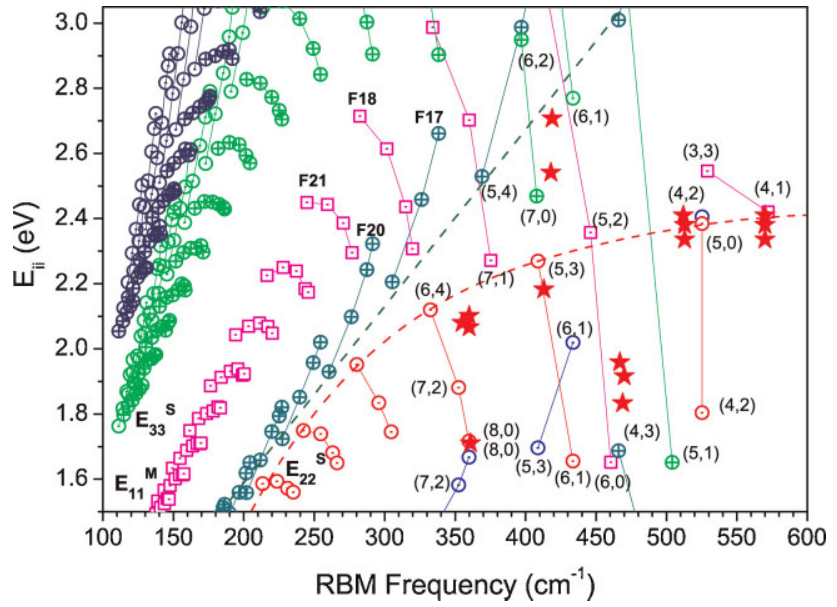

Figure 4 (online colour at: www.pss-b.com) Transition energies versus RBM frequency (Kataura-Popov plot) for SWCNTs with high and ultra-high curvature as obtained from extended tight binding calculations corrected for many electron contributions. Open and crossed circles are for SC type 1 and type 2 tubes. Open squares are for $E_{11}^{\mathrm{M}}$ transitions in metallic tubes. Family members are connected by lines. Asterisks are experimental results. A few selected families $(F)$ and a few selected tubes $[(n, m)]$ are indicated. The dashed lines are guides for the eye to follow $E_{22}^{\mathrm{SC}}$ transitions for the members of type 1 tubes with highest chiral angle (red) and similarly for the type 2 tubes (green).

Open circles represent SC type 1 tubes $(2 n+$ $m(\bmod 3)=1)$ whereas circles with crosses represent SC type 2 tubes $(2 n+m(\bmod 3)=2)$. Changes in transition energies are dramatic as for example for the tube $(6,1)$ of family 13 the $E_{11}^{\mathrm{SC}}$ transition occurs at a higher energy than the corresponding $E_{22}^{\mathrm{SC}}$ transition and for the $(6,0)$ tube of family 12 the $E_{11}^{\mathrm{M}}$ transition is down shifted to $1.65 \mathrm{eV}$ as compared to $5.3 \mathrm{eV}$ where it is expected from a simple tight binding calculation.

The experimental results can be inserted into this diagram as shown by the red asterisks. They match reasonably well to the calculated values and thus allow to assign chiralities to the inner tubes. A summary of the measured Raman lines together with the corresponding lasers used for excitation is given in Table 1.

The scaling factor was determined as to give the smallest root mean square value for the difference between experimentally and theoretically determined RBM frequencies. The assignment from Table 1 is identical to the assignment given in Ref. [16] except for the two highest frequencies. For these frequencies the anomalous relation between tube diameter and RBM frequency from the DFT calculation was considered. The assignment of the outer tube for the observed inner tube was obtained from adding twice the van der Waals distance to the observed inner tube diameters and looking for the best matching outer tube.

It is instructive to plot the data from Table 1 on a diagram with the product of RBM frequency and tube diameter versus inverse tube diameter. This is done in Fig. 5 where experiments are compared to calculations. 
Table 1 Experimental results for RBM frequencies $\omega$ and corresponding laser excitation energies (Col. 1, 2) compared to RBM frequencies calculated by DFT and reported in Ref. [12]. Transition energies are according to Plank et al. [16] (Col. 3, 4). The frequencies in Col. 3 are scaled by 0.98 to compensate for overestimation from DFT-LDA calculation. Column 5 has the chirality assignment and Col. 6 the tube diameter from DFT calculation.

\begin{tabular}{|c|c|c|c|c|c|}
\hline \multicolumn{2}{|c|}{ experiment } & \multicolumn{2}{|c|}{ calculated } & \multirow{2}{*}{$\frac{\text { assignment }}{(\mathrm{n}, \mathrm{m})}$} & \multirow{2}{*}{$\begin{array}{c}\text { diameter } \\
\begin{array}{c}d_{\mathrm{t}} \\
(\mathrm{nm})\end{array}\end{array}$} \\
\hline $\begin{array}{l}\omega \\
\left(\mathrm{cm}^{-1}\right)\end{array}$ & $\begin{array}{l}\text { laser } \\
(\mathrm{eV})\end{array}$ & $\begin{array}{c}\omega \\
\left(\mathrm{cm}^{-1}\right)\end{array}$ & $\begin{array}{l}E_{j j} \\
(\mathrm{eV})\end{array}$ & & \\
\hline 570 & 2.38 & 572.3 & 2.38 & $(4,1) @(11,4)$ & 0.3732 \\
\hline 513 & 2.38 & 525.3 & 2.40 & $(5,0) @(12,3)$ & 0.4043 \\
\hline 513 & 2.38 & 525.3 & 2.40 & $(4,2) @(10,6)$ & 0.4252 \\
\hline 470 & 1.83 & 466.5 & 1.69 & $(4,3) @(10,7)$ & 0.4833 \\
\hline 419 & 2.71 & 424.3 & 2.77 & $(6,1) @(13,4)$ & 0.5216 \\
\hline 417 & 2.54 & 404.2 & 2.47 & $(7,0) @(11,7)$ & 0.5546 \\
\hline 413 & 2.18 & 397.3 & 2.27 & $(5,3) @(7,11)$ & 0.5546 \\
\hline 363 & 1.71 & 351.3 & 1.88 & $(8,0) @(15,3)$ & 0.6319 \\
\hline 361 & 2.06 & 346.9 & 1.88 & $(7,2) @(13,6)$ & 0.6492 \\
\hline
\end{tabular}

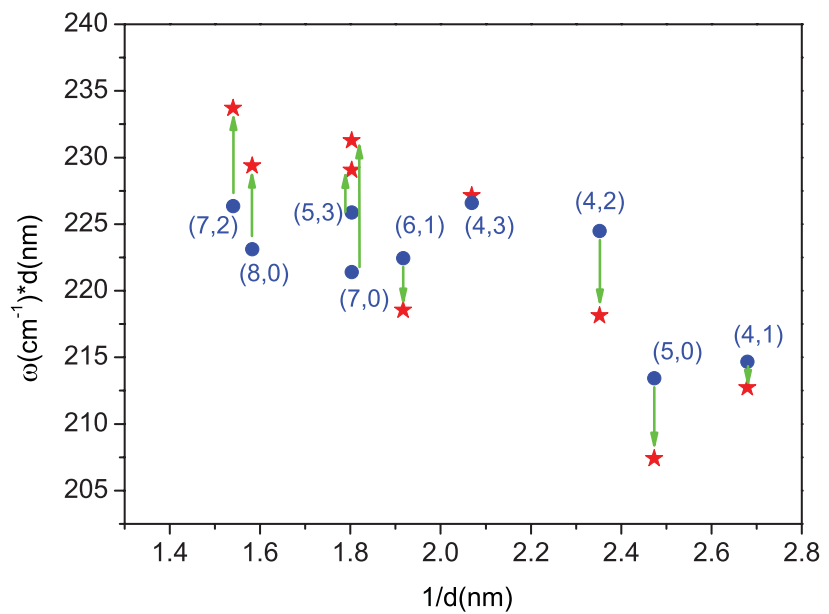

Figure 5 (online colour at: www.pss-b.com) Product of RBM frequency and DFT tube diameter versus inverse tube diameter. Blue bullets are as calculated, red asterisks are from the experiment. Green arrows indicate the difference.

The product on the ordinate is a measure how good the RBM frequencies follow a $C / d_{\mathrm{t}}$ law. Interestingly, while from the calculations for relative large tubes the product is close to constant it follows an almost linear decrease for decreasing tube diameters. In contrast, the experiments exhibit an almost linear decrease with inverse tube diameter. As a consequence there is a systematic deviation between experiment and calculation. For the small tubes the experimental values fall below the calculated values whereas for the larger tubes this trend is reversed.

4 Discussion and conclusion Transformation of FeCp ${ }_{2} @ S W C N T$ to DWCNTs was successfully demonstrated at temperatures considerably lower than the transformation of $\mathrm{C}_{60} @$ SWCNT to DWCNTs. This is in agreement with previous [17] and most recent experiments for DWCNTs grown from ferrocene [18] where transformation temperatures as low as $500{ }^{\circ} \mathrm{C}$ were observed. This means the growth process for the ultra-high curvature tubes is like in the previous cases catalytic and controlled by very small iron carbide $\left(\mathrm{Fe}_{3} \mathrm{C}\right)$ particles. Since the space available for this particle is only of the order of $0.5 \mathrm{~nm}$ and the unit cell of iron carbide is about $0.4 \times 0.5 \times 0.7 \mathrm{~nm}^{3}$ the catalytic reaction proceeds in our case by a particle with the size of a unit cell. This cell has only 4 carbon and 12 Fe atoms. With increasing growth of the inner tubes this particle becomes longer and longer as more and more iron carbide is generated but the cross-section remains confined to the unit cell size. Since the solubility of $\mathrm{C}$ in $\mathrm{Fe}_{3} \mathrm{C}$ is of the order of some percent the growth process can proceed only step by step with individual carbon atoms. Whether or not the catalytic particle retains its crystal structure is difficult to predict. On the one hand the particle is accommodated in a highly ordered environment of the outer tube carbons but on the other hand not even a medium long range order can be established.

The diameter of the smallest tubes observed is only $0.4 \mathrm{~nm}$ or just below, with a DFT-RBM frequency (unscaled) of $584 \mathrm{~cm}^{-1}$. It is not quite as small as predicted from molecular dynamical calculations. From subtracting two times the van der Waals distance of $0.34 \mathrm{~nm}$ from the smallest diameter which can accept a ferrocene molecule a DFT tube diameter for the inner tubes of $0.32 \mathrm{~nm}$ would be possible. This corresponds to a $(4,0)$ or to a $(3,2)$ tube with an RBM frequency of $627 \mathrm{~cm}^{-1}$. The simplest reason why this tube was not observed could be due to the fact that none of the lasers used here matched to any electronic transitions for such tubes. Alternatively and more likely the tube does not grow because the catalytic particle drops below the unit cell size and therefore catalytic activity is reduced.

Some of the predicted tubes were not observed in the experiments. This is most likely also due to the fact that none of the used lasers matched to a resonant transition. This may be so even if there were lasers close to the calculated transition energies from Fig. 4 since the precision of the calculation may be not better than $100 \mathrm{meV}$.

The line width for the response from the inner tubes is considerably larger than one would expect from previous measurements on DWCNTs. Since clean room conditions are the same in the case of DWCNT grown from HiPco or from conventional diameter tubes the reason for the enhanced line width may be due to a length distribution of inner tube segments. Molecular mechanical calculations did indeed show, that even in the optimum case only about $50 \%$ of the outer tubes can be filled with inner tubes and HRTEM revealed segmented inner tubes [16]. Since the RBM frequency depends on the tube length, inhomogeneous broadening can be responsible for enhanced line widths.

The RBM frequencies definitely do not follow a $C / d_{\mathrm{t}}$ law as can be seen from Fig. 5. This is consistent with the DFT 
calculations. The interesting behavior is the systematic deviations of the experiments to theory. This is of course partly due to the fact that measurements were done for tubes inside a master tube whereas calculations were performed for free tubes. However, as already mentioned, the effect of the tube-tube interaction may be small if the wall-to-wall distance is close to the van der Waals distance of $0.34 \mathrm{~nm}$. On the other hand, for very small tubes grown from unit cell sized catalytic particles, it may not be possible to adjust properly to the outer tube diameter and inner tube diameters end up smaller than corresponding to the van der Waals distance. As a consequence they will expand and their RBM frequency will be reduced below the value for the free tubes. In contrast, the larger diameter tubes adjust properly but feel the tube-tube interaction more strongly and therefore their RBM frequencies shift upwards. This is certainly also a consequence of their softer mode frequency compared to the very small diameter tubes. A detailed DFT calculations as it is presently on the way [10] will shine more light on this problem.

In conclusion, we have demonstrated that SWCNTs with ultra-high curvature can be grown from HiPco tubes with an approximate diameter of $1 \mathrm{~nm}$ if ferrocene is used as a carbon feedstock filled into the HiPco tubes. From the low transformation temperature a catalytic growth process is anticipated which, consequently operates for particles with dimensions down to the unit cell. Dramatic changes in the electronic structure as compared to tubes of conventional size were demonstrated from resonance Raman experiments. For the lower diameter end of the diameter distribution the tube diameters depict systematic deviations from the $C / d_{\mathrm{t}}$ law consistent with DFT calculations. Deviations form the DFT results are systematic and suggested to be a consequence of inner tube expansion for the smallest observed inner tubes and tube-tube interaction induced up shift for the larger set of inner tubes.

Acknowledgements Supported by the DFG project DFG PI 440/3 and by the FWF project I83-N20 (ESF IMPRESS). L. W. acknowledges funding by the French National Research Agency (ANR) project bl-inter09_482166. Valuable discussions with Th. Pichler are gratefully acknowledged.

\section{References}

[1] F. Kuemmeth, S. Ilani, D. C. Ralph, and P. L. McEuen, Nature 452 (7186), 448-452 (2008).

[2] D. Huertas-Hernando, F. Guinea, and A. Brataas, Phys. Rev. B 74, 155426 (2006).

[3] D. Connétable, G. M. Rignanese, J. C. Charlier, and X. Blase, Phys. Rev. Lett. 94, 015503-1-4 (2005).

[4] R. Lortza, Q. C. Zhang, W. Shi, J. T. Ye, C. Y. Qiu, Z. Wang, H. T. He, P. Sheng, T. Z. Qian, Z. K. Tang, N. Wang, X. X. Zhang, J. N. Wang, and C. T. Chan, Proc. Natl. Acad. Sci. USA 106(18), 7299-7303 (2009).

[5] C. D. Spataru, S. Ismail-Beigi, L. X. Benedict, and S. G. Louie, Phys. Rev. Lett. 92, 077402-1-4 (2004).

[6] E. Chang, G. Bussi, A. Ruini, and E. Molinari, Phys. Rev. Lett. 92, 196401 (2004).

[7] C. L. Kane and E. J. Mele, Phys. Rev. Lett. 93, 197402 (2004).

[8] A. Jorio, C. Fantini, M. A. Pimenta, R. B. Capaz, G. G. Samsonidze, G. Dresselhaus, M. S. Dresselhaus, J. Jiang, N. Kobayashi, A. Grüneis, and R. Saito, Phys. Rev. B 71, 075401 (2005).

[9] V. N. Popov, New J. Phys. 6, 17 (2004).

[10] L. Wirtz, (unpublished), Radial breathing modes of doublewalled carbon nanotubes from DFT calculation.

[11] V. N. Popov and L. Henrard, Phys. Rev. B 65, 235415 (2002).

[12] J. Kürti, V. Zólyomi, M. Kertesz, and G. Sun, New J. Phys. 5, 125 (2003).

[13] X. Gonze, J. M. Beuken, R. Caracas, F. Detraux, M. Fuchs, G. M. Rignanese, L. Sindic, M. Verstraete, G. Zerah, F. Jollet, M. Torrent, A. Roy, M. Mikami, P. Ghosez, J. Y. Raty, and D. Allan, Comput. Mater. Sci. 25, 478 (2002).

[14] R. Pfeiffer, H. Kuzmany, C. Kramberger, C. Schaman, T. Pichler, H. Kataura, Y. Achiba, J. Kürti, and V. Zólyomi, Phys. Rev. Lett. 90, 225501-1-4 (2003).

[15] H. Telg, J. Maultzsch, S. Reich, F. Hennrich, and C. Thomsen, Phys. Rev. Lett. 93, 177401 (2004).

[16] W. Plank, R. Pfeiffer, C. Schaman, H. Kuzmany, M. Calvarasi, F. Zerbetto, and J. Meyer, ACS Nano 4, 4515-4522 (2010).

[17] H. Shiozawa, T. Pichler, A. Grüneis, R. Pfeiffer, H. Kuzmany, Z. Liu, K. Suenaga, and H. Kataura, Adv. Mater. 20(8), 1443 (2008).

[18] H. Shiozawa, C. Kramberger, R. Pfeiffer, H. Kuzmany, T. Pichler, Z. Liu, K. Suenaga, H. Kataura, and S. R. P. Silva, Adv. Mater. (2010), published online DOI 10. 1002/adma. 201001211. 